\title{
Oblique polarized reflectance spectroscopy for depth sensitive measurements in the epithelial tissue
}

\author{
Maria K. Jimenez ${ }^{\mathrm{a}}$, Leonid Fradkin ${ }^{\mathrm{a}}$, Linda T. Nieman ${ }^{\mathrm{b}}$, Sylvia Lam ${ }^{\mathrm{c}}$, Catherine Poh $^{\mathrm{c}}$, Konstantin \\ Sokolov ${ }^{\mathrm{a}, \mathrm{b}}$ \\ ${ }^{a}$ Department of Biomedical Engineering, The University of Texas at Austin, 107 West Dean Keeton \\ Street, Austin, TX 78712; ${ }^{\mathrm{b}}$ Department of Imaging Physics, The University of Texas M.D. Anderson \\ Cancer Center, 1515 Holcombe Boulevard, Houston, TX 77030; 'Integrative Oncology Department, \\ British Columbia Cancer Agency, 675 West $10^{\text {th }}$ Avenue, Vancouver, British Columbia, V5Z 1L3, \\ Canada
}

\begin{abstract}
Optical spectroscopy has shown potential as a tool for precancer detection by discriminating alterations in the optical properties within epithelial tissues. Identifying depth-dependent alterations associated with the progression of epithelial cancerous lesions can be especially challenging in the oral cavity due to the variable thickness of the epithelium and the presence of keratinization. Optical spectroscopy of epithelial tissue with improved depth resolution would greatly assist in the isolation of optical properties associated with cancer progression. Here, we report a fiber optic probe for oblique polarized reflectance spectroscopy (OPRS) that is capable of depth sensitive detection by combining the following three approaches: multiple beveled fibers, oblique collection geometry, and polarization gating. We analyze how probe design parameters are related to improvements in collection efficiency of scattered photons from superficial tissue layers and to increased depth discrimination within epithelium. We have demonstrated that obliquely-oriented collection fibers increase both depth selectivity and collection efficiency of scattering signal. Currently, we evaluate this technology in a clinical trial of patients presenting lesions suspicious for dysplasia or carcinoma in the oral cavity. We use depth sensitive spectroscopic data to develop automated algorithms for analysis of morphological and architectural changes in the context of the multilayer oral epithelial tissue. Our initial results show that OPRS has the potential to improve the detection and monitoring of epithelial precancers in the oral cavity.
\end{abstract}

Keywords: elastic light scattering, polarized light, reflectance spectroscopy, oral cancer diagnosis

\section{INTRODUCTION}

More than $80 \%$ of all cancers originate in the tissue lining organs known as epithelium. ${ }^{1}$ Epithelial tissue is avascular and makes up the first $25-500 \mu \mathrm{m}$ of tissue depending on the organ site. Conversely, the underlying stromal layer is primarily composed of collagen and contains fibroblast cells and blood vessels. Subsurface tissue alterations associated with the concurrent changes in the epithelial and stromal tissue layers are important in early detection and diagnosis of epithelial cancer. In normal tissue, the epithelium and stroma form two distinct layers. Benign lesions typically have an additional keratin layer. Moreover, epithelial tissue becomes thickened from the over proliferation of basal cells. Finally, as tissue becomes cancerous, the layered organization within epithelium along with the distinct boundary between the epithelium and underlying stroma vanishes. ${ }^{2}$ Widespread noninvasive clinical imaging modalities such as ultrasound, MRI, and CT do not offer adequate resolution or contrast to detect these early alterations within epithelial pre-cancers ${ }^{3}$ whereas optical methods have shown great promise detecting local changes in tissue morphology and biochemistry associated with precancer. ${ }^{4-12}$ In particular, depth-sensitive optical spectroscopy has shown immense potential as a tool for precancer detection by discriminating alterations in the optical properties within epithelial tissues.

Identifying depth-dependent alterations associated with the progression of epithelial cancerous lesions is challenging due to the inherent turbidity of tissue. While the epithelium is thin and optically transparent, the vascularized stroma underneath is thick and turbid which causes spectroscopic signals to be dominated by the scattering and hemoglobin absorption from the stroma. To overcome this issue, several methods aim to improve signal collection from the superficial epithelial layer in reflectance spectroscopy measurements by using a few low cost elements. Approaches based on varying the standard probe geometry where all optical fibers are normal to the tissue surface achieves depth

Biomedical Applications of Light Scattering VII, edited by Adam P. Wax, Vadim Backman, Proc. of SPIE Vol. 8592, 859203 · C 2013 SPIE · CCC code: 1605-7422/13/\$18 doi: $10.1117 / 12.2004875$

Proc. of SPIE Vol. $8592859203-1$ 
sensitivity with a variable aperture ${ }^{13,}{ }^{14}$, variable source-detector separations, ${ }^{14-17}$ or with differential path length spectroscopy. ${ }^{18}$ Other approaches to improve depth sensitivity in fiber optic probes include a spherical lens ${ }^{19-21}$ and employing an oblique orientation to the fibers in order to favorably collect either superficial or deep penetrating photons. ${ }^{22-26}$ Our group along with others have developed a polarization gating scheme to separate the epithelial signal from the diffuse background signal of the underlying stromal layer. ${ }^{27-33}$ Furthermore, to achieve the discrimination of optical signal within epithelial tissue with improved depth sensitivity, our laboratory developed a fiber optic probe for oblique polarized reflectance spectroscopy (OPRS). ${ }^{2}$ The OPRS probe is capable of depth sensitive detection of optical signal by combining oblique collection geometry with polarization gating.

While these methods are promising, they do not account for the variable thickness of the epithelium that may result from disease progression, benign inflammatory conditions, and intra- or inter-patient variation. Ideally, a depth sensitive fiber optic probe would accommodate changes in epithelial thickness while effectively discriminating scattering signals from different tissue layers. To achieve improved depth resolution to accommodate for variable epithelial thickness, we developed an OPRS probe with multiple beveled collection fibers to instantaneously interrogate several depths within tissue. $^{34}$

\section{MATERIALS AND METHODS}

\subsection{Instrumentation}

A schematic of the OPRS system is shown Figure 1(A). Light from a $20 \mathrm{~W}$ tungsten halogen broadband light source (Ocean Optics, HL2000HP-FHSA) is delivered via an optical fiber. The proximal ends of additional collection fibers are coupled to a spectrometer (PI Acton SpectraPRO SP-2356, Pixis 2KB) which uses $150 \mathrm{~g} / \mathrm{mm}$ grating optimized for visible wavelength (500 nm blaze). The spectrometer disperses the light from each collection fiber simultaneously onto an imaging CCD. Figure 1(B) shows the distal end of the optical probe and Figure 1(C) displays an image of the constructed probe in a fluorescent medium to demonstrate the overlap of the illumination beams and the collection field of views. The fiber optic probe is comprised of a beveled fiber (BF) array. All the fibers are identical with a silica core $(\mathrm{n}=1.458)$ and fluorine doped cladding $(\mathrm{n}=1.455)$ with diameters of $100 \mu \mathrm{m}$ and $110 \mu \mathrm{m}$, respectively, and a NA of 0.12 (CeramOptec Industries, Inc., WF 100/110 P12). The collection fibers are labeled according to their distance from the illumination fiber wherein BF1 has one fiber diameter separation from the illumination fiber, BF2 has two fiber diameters separation from the illumination fibers, and so on. Fibers farther from the illumination beam sample deeper tissue regions. The beveled collection fibers are obliquely polished at an angle of $40^{\circ}$ with respect to the tissue plane which allows fibers to be oriented along the same axis normal to the tissue surface as oppose an oblique orientation. A $150 \mu \mathrm{m}$ thick polarizing film $(\mathrm{n}=1.458)$ with a low extinction transmittance of cross-polarized light $(0.002 \%)$ is secured to the distal end of optical fibers and is used to define the polarization states of the illumination and collection channels. In this paper, light collected with polarization parallel to the illumination polarization, i.e. co-polarized, is defined as parallel light whereas light collected with polarization perpendicular, or cross-polarized, with respect to the illumination polarization is defined as perpendicular. Then, a $200 \mu \mathrm{m}$ quartz window $(\mathrm{n}=1.54)$ is fixed to the end of the probe to protect the assembly, maintain the air gap between the fibers and tissue, and achieve an optimal sampling depth. Images of the constructed probe are shown in Figure 2. 

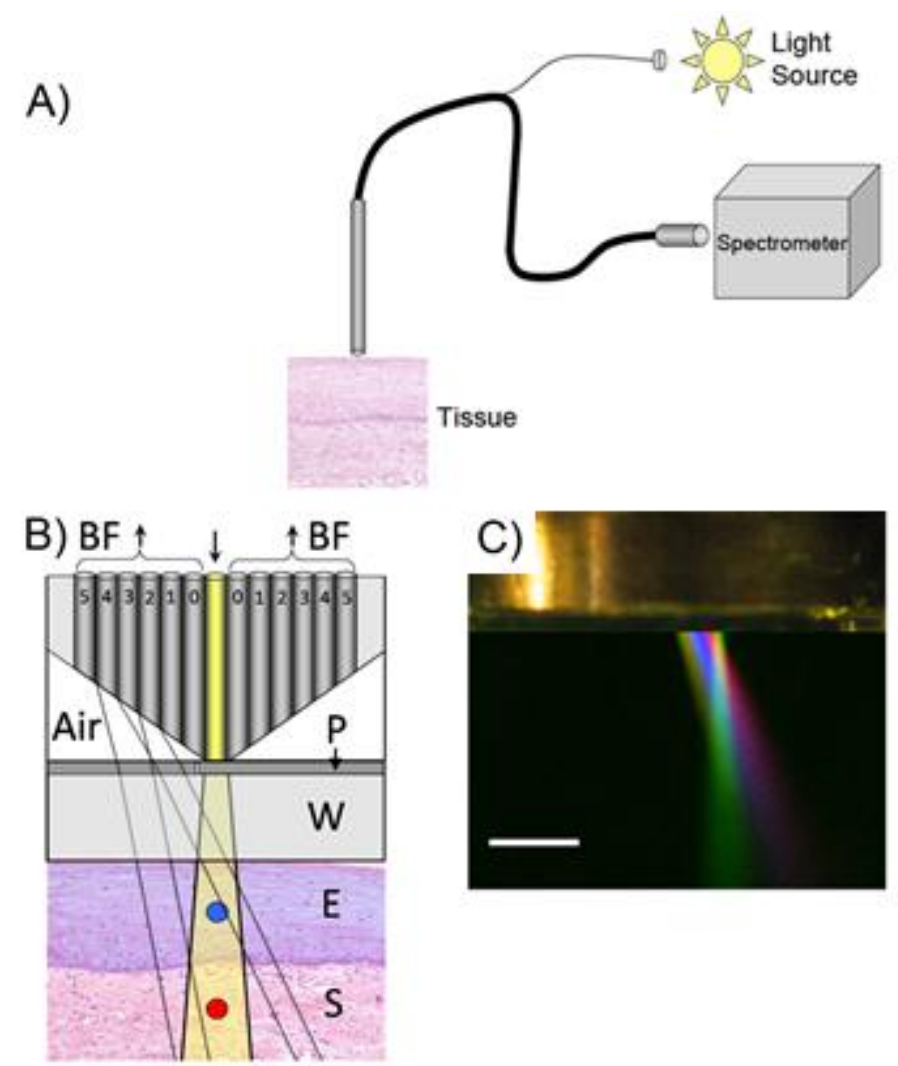

Figure 1. (A) Schematic of the overall OPRS system. (B) Distal end of optical probe with multiple beveled collection fibers for the simultaneous measurements of several depths. The $\mathrm{P}$ signifies the polarizing film, $\mathrm{W}$ is the probe window, and $\mathrm{E}$ and $\mathrm{S}$ represent the epithelium and stromal tissue layer, respectively. (C) Picture of the distal ends of the constructed probe in fluorescing medium to show the areas of overlap between the illumination and collection cones. Scale bar is $1 \mathrm{~mm}$. Modified from references [34].
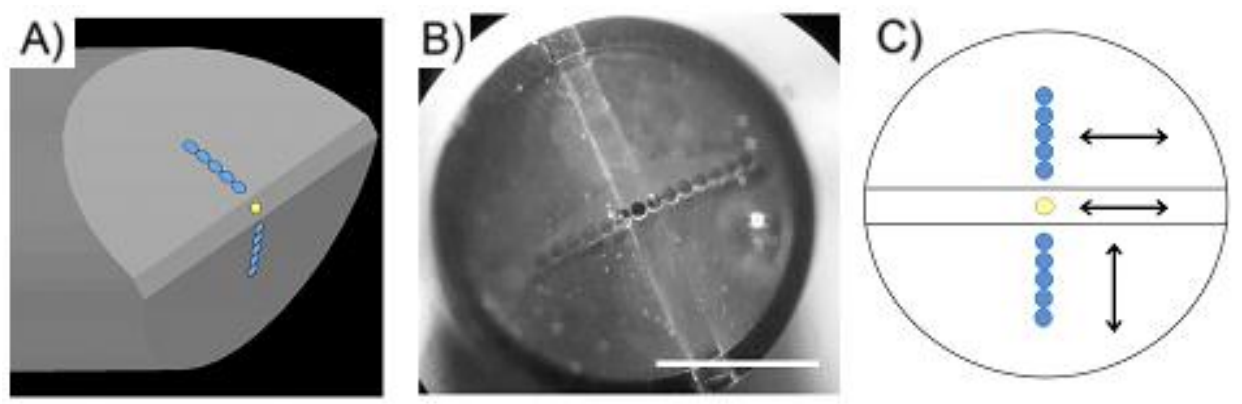

Figure 2. (A) 3D rendering of the beveled multi-fiber OPRS probe design. (B) Actual image of the constructed probe without the polarizing film and protective window with the illumination fiber in focus. Scale bar is $1 \mathrm{~mm}$ (C) Orientation of the polarization transmission axis for the multiple beveled fiber (BF) arrays.

\subsection{Tissue phantoms}

Although fiber optic probe designs are ultimately validated using real tissue, tissue-simulating phantoms provide a convenient tool to characterize the performance of fiber probes in a scattering media in a controlled and reproducible manner. ${ }^{36,37}$ Using considerations from Mie theory, the concentration and size of beads can be selected to match the scattering conditions of normal tissue. Similarly, a more complex three-layer model can be constructed matching the 
scattering conditions found in layered epithelium such as within pre-cancerous tissue. Use of tissue phantoms allows the assessment of the signal contribution from each layer under well-defined scattering conditions that can be adjusted by varying phantom composition, e.g. size and concentration of polystyrene beads. We tested the capability of a beveled multi-fiber OPRS probe design to interrogate tissue scattering properties with depth discrimination using a three-layer bead phantom: top $-5.01+/-0.14 \mu \mathrm{m}(\mathrm{g}=0.89)$, middle $-8.31+/-0.66 \mu \mathrm{m},(\mathrm{g}=0.92)$, and bottom $-2.50+/-0.16 \mu \mathrm{m}(\mathrm{g}$ $=0.83$ ). All values are reported at $550 \mathrm{~nm}$. The top layer mimics normal epithelium with a scattering coefficient of 33

$\mathrm{cm}^{-1}$, the middle layer mimics a pre-cancerous growth with a reduced scattering coefficient of $71 \mathrm{~cm}^{-1}$, and, finally, the bottom layer represents the highly scattering stroma with a reduced scattering coefficient $189 \mathrm{~cm}^{-1} .34$

The challenge with measurements of layered tissue lies in the determination of the scattering signal contribution from each independent layer. With a polystyrene bead phantom, this can be accomplished by taking advantage of the unique size-dependent oscillatory scattering spectrum produced by beads, and using a single uniform bead size for each layer. Thus, a three-layer bead phantom provides a simple way to validate the ability of a probe to separate scattering from multiple layers. The scattering spectrum for each bead size and refractive index is calculated precisely using Mie theory. The measured spectrum from a three-layer bead phantom is a mixture of the oscillatory signals from each layer, but can be decomposed into its constituent parts using a fast Fourier Transform (FFT). A Hann window was used to eliminate spectral leakage and maintain proportionality between the FFT amplitude and the original signal.

\subsection{Clinical measurement}

A clinical study is being conducted with informed consent on patients over the age of 18 that are referred to the Department of Integrative Oncology at the British Columbia Cancer Agency (BCCA) with oral mucosa lesions suspicious for dysplasia or carcinoma. A standard oral cavity examination is performed followed by spectroscopic measurements. The sample sites are assessed for three distinct diagnostic categories: normal, benign, and dysplasia. Calibration spectra are acquired before each patient evaluation using a diffuse reflectance substrate standard (SRS-99, Labsphere, Inc.). The OPRS probe in the clinical studies has three pairs of beveled detector fibers. Each pair collects light with polarization parallel and perpendicular to the polarization of the illumination light.

\subsection{Data processing}

The collected parallel and perpendicular spectra are dark subtracted using the spectra from a minimally reflecting black substrate (SRS-02, Labsphere, Inc.) to account for the total internal reflections within the optical fibers and subsequently divided by the spectra obtained from the diffuse reflectance substrate standard to account for the spectral profile of the source and the wavelength-dependent response of the detection system. Then, the patient data are normalized by a previously obtained depth profile curve. The depth profile curves were obtained using a diffuse white scatterer composed of a white Teflon tape ( $c a .90 \mu \mathrm{m}$ thick). For each collection fiber, the signal intensity (integrated over all wavelengths) was measured and plotted at probe-tape separations of 0-3000 $\mu \mathrm{m}$. Dividing the patient data by the integral of the depth profile curves accounted for differences in the collection efficiencies between fiber pairs that are equidistant from the illumination source fiber. The processed parallel and perpendicular spectra were then analyzed.

\section{RESULTS}

\subsection{Validation of depth sensitivity in tissue phantoms}

To demonstrate that an array of multiple oblique fibers can interrogate tissue at different depths we carried out initial measurements in the three-layer bead phantom with a beveled multi-fiber probe without polarization gating. Figure 3 shows the measured FFT amplitudes of the top, middle, and bottom layers extracted from the scattering spectrum of a three-layer bead phantom. The results show that even without polarization sensitivity, the beveled multi-fiber probe design is capable of discriminating sub-regions within a phantom mimicking pre-cancerous tissue. The collection fibers closest to the illumination fiber have highest relative collection efficiency from the top layers of the phantom. Hence, within the top phantom layer, BF0 has the greatest collection efficiency. Within the middle layer, BF2 and BF3 start to collect appreciable signal. And lastly, the bottom layer is most efficiently interrogated by BF2. This provides evidence that the beveled multi-fiber probe design can be effective for depth sensitive measurements under scattering conditions. 
The probe used for the unpolarized phantom measurements had a reference FTF that was directly adjacent to the illumination fiber. ${ }^{34}$ A FTF measurement permits direct comparison of a standard FTF probe geometry with the beveled multi-fiber geometry. Figure 3 demonstrates that for all layers of the phantom, beveled fibers BF0-BF3 have significantly better collection efficiency than the FTF. For the top layer, the signal collection improves by $4000 \%$ for $\mathrm{BF} 0$ to $140 \%$ for $\mathrm{BF} 3$ and by $1500 \%$ for $\mathrm{BF} 0$ to $60 \%$ for $\mathrm{BF} 3$ for the middle layer.
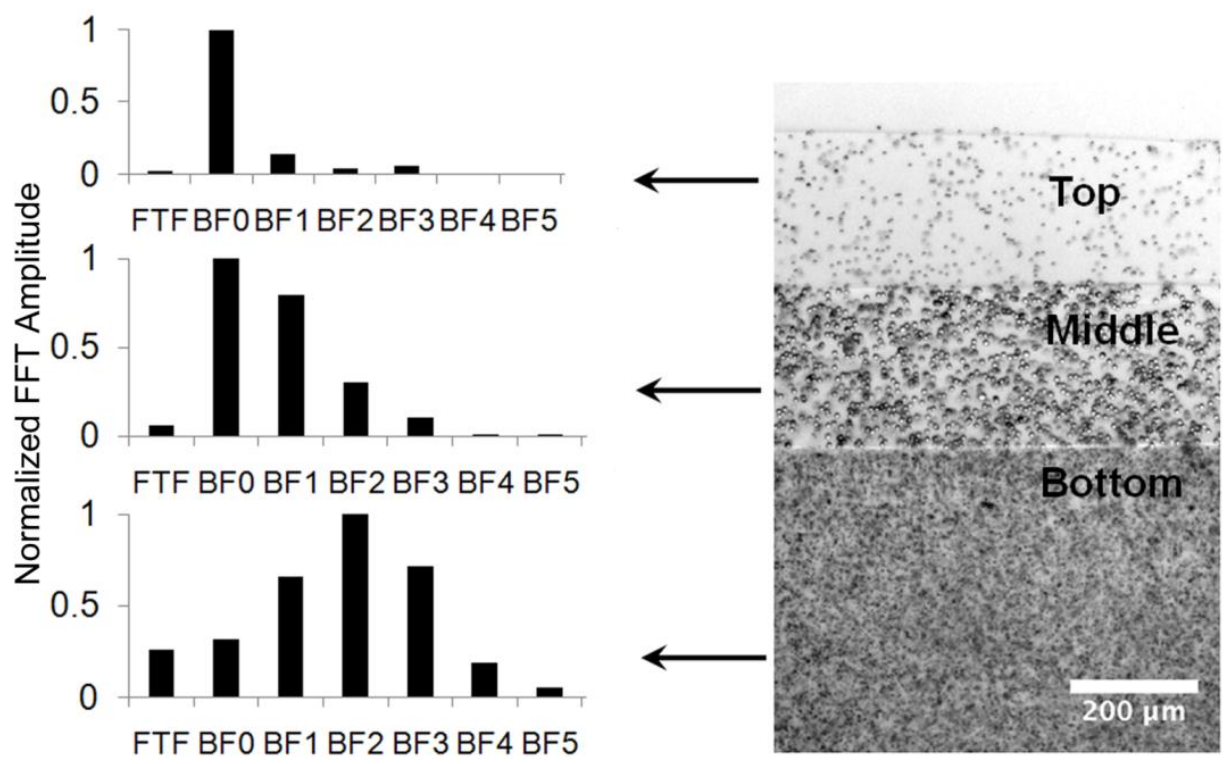

Figure 3. Evaluation of the beveled multi-fiber probe design in a three-layer phantom. The normalized fast Fourier transform (FFT) amplitudes of unpolarized light from each layer of the three-layer bead phantom shown on the right. The bar graphs show the collection efficiency of the beveled fibers (BF) for each phantom layer. The panel on the right is a transmittance image of a cross-sectional section of the corresponding three-layer bead phantom.

\subsection{In vivo measurements}

We have collected OPRS spectra from 18 patients and 65 sites. Figure 4 shows an example from a patient with a severe dysplasia (SD) in the oral cavity.

The dips association with hemoglobin absorption can clearly be observed at $540 \mathrm{~nm}$ and $580 \mathrm{~nm}$. As expected for each BF pair, the parallel signal is greater in intensity than its perpendicular counterpart. A qualitative look at the spectra reveals clear differences between the OPRS measurements from normal, benign and severe dysplasia (SD). For each of the parallel fibers, the SD spectra have larger overall intensities than the normal spectra; these differences can be associated with higher scattering from the dysplastic epithelium due to morphological changes such as increased nuclear size, hyperchromasia and pleomorphism. ${ }^{1}$ Conversely, the perpendicular fibers have SD curves that are lower in intensity than the normal curves; this effect can be associated with loss of collagen fiber density in the stroma during malignant transformation. ${ }^{8}$ One of the most clinically challenging classification undertakings involves the ability to distinguish dysplastic tissue from benign tissue, which has the outward appearance of dysplasia or carcinoma. Therefore, it is encouraging that the obtained results show several clear separations between benign and SD sites. The clinical study is ongoing and we continue to collect more data. As we increase our sample size, we hope to achieve sensitivity and specificity that exceed our previous values obtained using an oblique polarized reflectance spectroscopy with two collection fibers. ${ }^{2}$ 

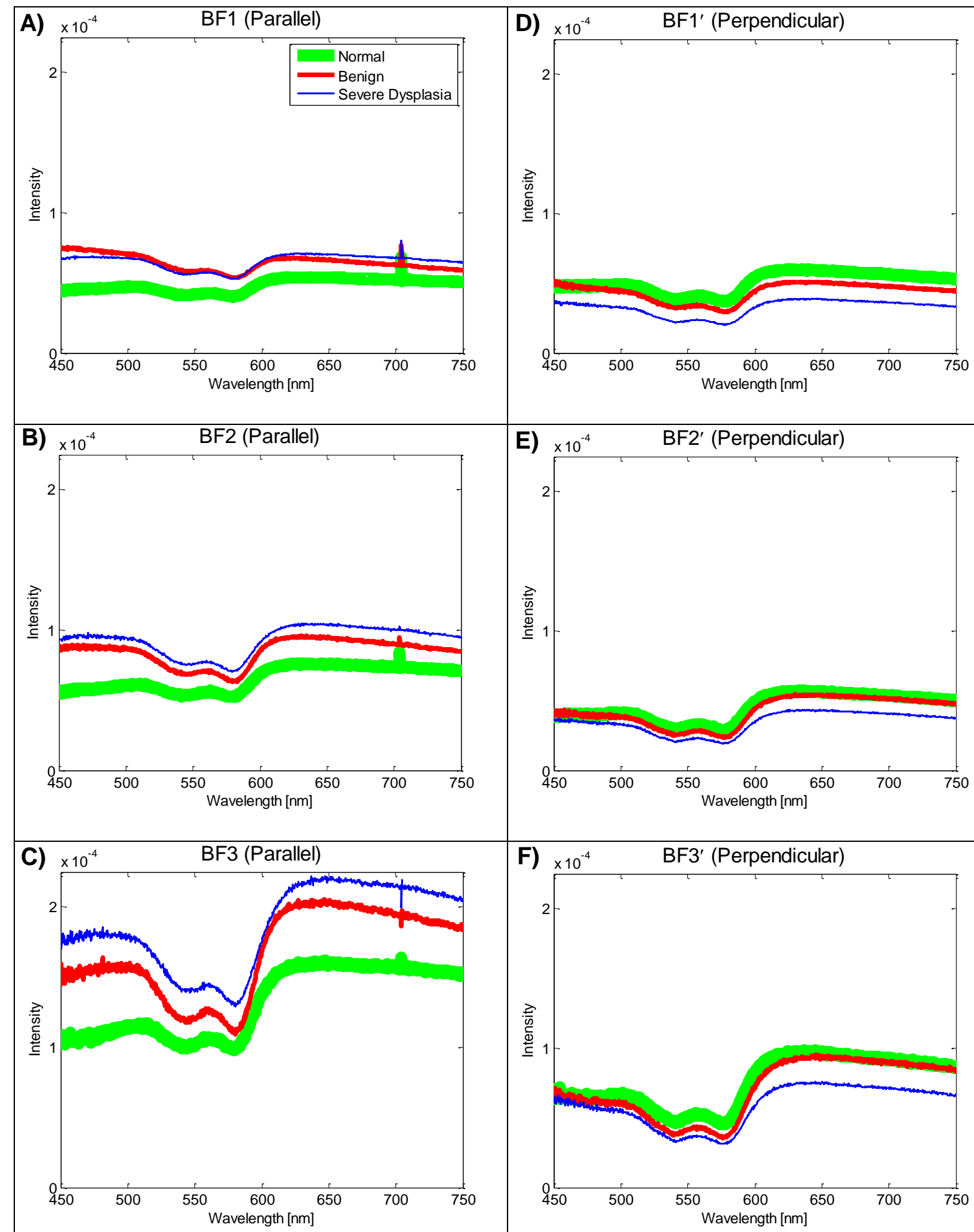

Figure 4: Examples of experimental OPRS spectra for each individual parallel (A-C) and perpendicular (D-F) collection fiber. The measurements are taken from the same patients. The measured sites were classified as normal, benign, and severe dysplasia. 


\section{CONCLUSIONS}

Since pre-cancer development is associated with concomitant changes in different layers of the epithelium and the stroma, it is vital from a clinical perspective to be able to extract optical signatures from several depths simultaneously. ${ }^{1}$

${ }^{2,8}$ However, measuring these changes in vivo can prove difficult due to variations in epithelial thickness caused by patient-to-patient variations or benign growths such as keratin which make spectroscopic interrogation of epithelial sublayers, and differentiation of scattering between the epithelium and the underlying stroma challenging. We believe OPRS has the potential to overcome these diagnostic challenges. The use of multiple BFs enables the simultaneous interrogation of multiple relative depths within tissue, thereby facilitating the observation of alterations within epithelial sublayers and allowing correction for variations in epithelial thickness. Furthermore, our initial results from a clinical trial demonstrate the potential of OPRS as a tool for the diagnosis and monitoring of oral premalignancies and malignancies.

[1] Cotran, R.S., V. Kumar, and T. Collins, [Pathologic basis of disease.]. 6th ed, Philadelphia: Saunders (1999).

[2] Nieman, L.T., C.-W. Kan, A. Gillenwater, M.K. Markey, and K. Sokolov, "Probing local tissue changes in the oral cavity for early detection of cancer using oblique polarized reflectance spectroscopy: a pilot clinical trial," Journal of Biomedical Optics 13(2), 024011 (2008).

[3] Flusberg, B.A., E.D. Cocker, W. Piyawattanametha, J.C. Jung, E.L.M. Cheung, and M.J. Schnitzer, "Fiber-optic fluorescence imaging," Nat Meth 2(12), 941-950 (2005).

[4] Richards-Kortum, R. and E. Sevick-Muraca, "Quantitative optical spectroscopy for tissue diagnosis," Annu. Rev. Chem. 47, 555-606 (1996).

[5] Bigio, I.J. and J.R. Mourant, "Ultraviolet and visible spectroscopies for tissue diagnostics: fluorescence spectroscopy and elastic-scattering spectroscopy," Physics in Medicine and Biology 42, 803-814 (1997).

[6] Wagnieres, G.A., W.M. Star, and B.C. Wilson, "In vivo fluorescence spectroscopy and imaging for oncological applications," Photochemistry and Photobiology 68, 603-632 (1998).

[7] Ramanujam, N., "Fluorescence spectroscopy of neoplastic and non-neoplastic tissues," Neoplasia (New York, N.Y.) 2(1-2), 89-117 (2000).

[8] Sokolov, K., M. Follen, and R. Richards-Kortum, "Optical spectroscopy for detection of neoplasia," Current Opinion in Chemical Biology 6(5), 651-658 (2002).

[9] Georgakoudi, I., "The color of cancer," Journal of Luminescence 119, 75-83 (2006).

[10] Ramachandran, J., T.M. Powers, S. Carpenter, A. Garcia-Lopez, J.P. Freyer, and J.R. Mourant, "Light scattering and microarchitectural differences between tumorigenic and non-tumorigenic cell models of tissue," Optics Express 15(7), 4039-4053 (2007).

[11] Georgakoudi, I., E.E. Sheets, M.G. Muller, V. Backman, C.P. Crum, K. Badizadegan, R.R. Dasari, and M.S. Feld, "Trimodal spectroscopy for the detection and characterization of cervical precancers in vivo," American Journal of Obstetrics and Gynecology 186(3), 374-382 (2002).

[12] Muller, M.G., T.A. Valdez, I. Georgakoudi, V. Backman, C. Fuentes, S. Kabani, N. Laver, Z. Wang, C.W. Boone, R.R. Dasari, S.M. Shapshay, and M.S. Feld, "Spectroscopic detection and evaluation of morphologic and biochemical changes in early human oral carcinoma," Cancer 97(7), 1681-1692 (2003).

[13] Liu, Q. and N. Ramanujam, "Relationship between depth of a target in a turbid medium and fluorescence measured by a variable-aperture method," Optics Letters 27(2), 104-106 (2002).

[14] Pfefer, T.J., L.S. Matchette, A.M. Ross, and M.N. Ediger, "Selective detection of fluorophore layers in turbid media: the role of fiber-optic probe design," Optics Letters 28(2), 120-122 (2003).

[15] Pfefer, T.J., K.T. Schomacker, M.N. Ediger, and N.S. Nishioka, "Multiple-fiber probe design for fluorescence spectroscopy in tissue," Applied Optics 41(22), 4712-4721 (2002).

[16] Changfang, Z., L. Quan, and N. Ramanujam, "Effect of fiber optic probe geometry on depth-resolved fluorescence measurements from epithelial tissues: a Monte Carlo simulation," Journal of Biomedical Optics $8(2), 237-247$ (2003).

[17] Mourant, J.R., T. Fuselier, J. Boyer, T.M. Johnson, and I.J. Bigio, "Predictions and measurements of scattering and absorption over broad wavelength ranges in tissue phantoms," Applied Optics 36(4), 949-957 (1997).

[18] Amelink, A., H.J.C.M. Sterenborg, M.P.L. Bard, and S.A. Burgers, "In vivo measurement of the local optical properties of tissue by use of differential path-length spectroscopy," Optics Letters 29(10), 1087-1089 (2004). 
[19] Schwarz, R.A., D. Arifler, S.K. Chang, I. Pavlova, I.A. Hussain, V. Mack, B. Knight, R. Richards-Kortum, and A.M. Gillenwater, "Ball lens coupled fiber-optic probe for depth-resolved spectroscopy of epithelial tissue," Optics Letters 30(10), 1159-1161 (2005).

[20] Scepanovic, O.R., Z. Volynskaya, C.R. Kong, L.H. Galindo, R.R. Dasari, and M.S. Feld, "A multimodal spectroscopy system for real-time disease diagnosis," Review of Scientific Instruments 80(4), 9 (2009).

[21] Schwarz, R.A., W. Gao, C.R. Weber, C. Kurachi, J.J. Lee, A.K. El-Naggar, R. Richards-Kortum, and A.M. Gillenwater, "Noninvasive Evaluation of Oral Lesions Using Depth-sensitive Optical Spectroscopy," Cancer 115(8), 1669-1679 (2009).

[22] Nieman, L., A. Myakov, J. Aaron, and K. Sokolov, "Optical sectioning using a fiber probe with an angled illumination-collection geometry: evaluation in engineered tissue phantoms," Applied Optics 43(6), 1308-1319 (2004).

[23] Wang, A.M.J., J.E. Bender, J. Pfefer, U. Utzinger, and R.A. Drezek, "Depth-sensitive reflectance measurements using obliquely oriented fiber probes," Journal of Biomedical Optics 10(4), 44017-41 (2005).

[24] Arifler, D., R.A. Schwarz, S.K. Chang, and R. Richards-Kortum, "Reflectance spectroscopy for diagnosis of epithelial precancer: model-based analysis of fiber-optic probe designs to resolve spectral information from epithelium and stroma," Applied Optics 44(20), 4291-4305 (2005).

[25] Reif, R., O. A'Amar, and I.J. Bigio, "Analytical model of light reflectance for extraction of the optical properties in small volumes of turbid media," Applied Optics 46(29), 7317-7328 (2007).

[26] Garcia-Uribe, A., N. Kehtarnavaz, G. Marquez, V. Prieto, M. Duvic, and L.H.V. Wang, "Skin cancer detection by spectroscopic oblique-incidence reflectometry: classification and physiological origins," Applied Optics 43(13), 2643-2650 (2004).

[27] Sokolov, K., R. Drezek, K. Gossage, and R. Richards-Kortum, "Reflectance spectroscopy with polarized light: is it sensitive to cellular and nuclear morphology," Optics Express 5(13), 302-317 (1999).

[28] Backman, V., R. Gurjar, K. Badizadegan, I. Itzkan, R.R. Dasari, L.T. Perelman, and M.S. Feld, "Polarized light scattering spectroscopy for quantitative measurement of epithelial cellular structures in situ," IEEE Journal of Selected Topics in Quantum Electronics 5(4), 1019-1026 (1999).

[29] Anderson, R.R., "Polarized light examination and photography of the skin," Archives Of Dermatology 127(7), 1000-1005 (1991).

[30] Jacques, S.L., J.R. Roman, and K. Lee, "Imaging superficial tissues with polarized light," Lasers in surgery and medicine 26, 119-129 (2000).

[31] Liu, Y., Y. Kim, X. Li, and V. Backman, "Investigation of depth selectivity of polarization gating for tissue characterization," Optics Express 13(2), 601-611 (2005).

[32] Demos, S.G. and R.R. Alfano, "Temporal gating in highly scattering media by the degree of optical polarization," Optics Letters 21(2), 161-163 (1996).

[33] Morgan, S.P. and M.E. Ridgway, "Polarization properties of light backscattered from a two layer scattering medium," Optics Express 7(12), 395-402 (2000).

[34] Nieman, L.T., M. Jakovljevic, and K. Sokolov, "Compact beveled fiber optic probe design for enhanced depth discrimination in epithelial tissues," Optics Express 17(4), 2780-2796 (2009).

[35] Sokolov, K., L.T. Nieman, A. Myakov, and A. Gillenwater, "Polarized reflectance spectroscopy for pre-cancer detection," Technology in Cancer Research \& Treatment 3(1), 1-14 (2004).

[36] Sokolov, K., J. Galvan, A. Myakov, A. Lacy, R. Lotan, and R. Richards-Kortum, "Realistic three-dimensional epithelial tissue phantoms for biomedical optics," Journal Of Biomedical Optics 7(1), 148-156 (2002).

[37] Liu, Y., Y.L. Kim, and V. Backman, "Development of a bioengineered tissue model and its application in the investigation of the depth selectivity of polarization gating," Applied Optics 44(12), 2288-2299 (2005). 